\title{
On the limits of Köhler activation theory: how do collision and coalescence affect the activation of aerosols?
}

\author{
Fabian Hoffmann \\ Institute of Meteorology and Climatology, Leibniz Universität Hannover, Hannover, Germany \\ Correspondence to: Fabian Hoffmann (hoffmann@muk.uni-hannover.de)
}

Received: 10 February 2017 - Discussion started: 15 February 2017

Revised: 8 June 2017 - Accepted: 13 June 2017 - Published: 10 July 2017

\begin{abstract}
Activation is necessary to form a cloud droplet from an aerosol, and it is widely accepted that it occurs as soon as a wetted aerosol grows beyond its critical radius. Traditional Köhler theory assumes that this growth is driven by the diffusion of water vapor. However, if the wetted aerosols are large enough, the coalescence of two or more particles is an additional process for accumulating sufficient water for activation. This transition from diffusional to collectional growth marks the limit of traditional Köhler theory and it is studied using a Lagrangian cloud model in which aerosols and cloud droplets are represented by individually simulated particles within large-eddy simulations of shallow cumuli. It is shown that the activation of aerosols larger than $0.1 \mu \mathrm{m}$ in dry radius can be affected by collision and coalescence, and its contribution increases with a power-law relation toward larger radii and becomes the only process for the activation of aerosols larger than $0.4-0.8 \mu \mathrm{m}$ depending on aerosol concentration. Due to the natural scarcity of the affected aerosols, the amount of aerosols that are activated by collection is small, with a maximum of 1 in 10000 activations. The fraction increases as the aerosol concentration increases, but decreases again as the number of aerosols becomes too high and the particles too small to cause collections. Moreover, activation by collection is found to affect primarily aerosols that have been entrained above the cloud base.
\end{abstract}

\section{Introduction}

Activation is necessary for the formation of droplets from aerosols. Accordingly, activation controls the number and size of cloud droplets and hence so-called aerosol-cloud in- teractions, e.g., cloud albedo (Twomey, 1974) or cloud lifetime (Albrecht, 1989). In contrast to cloud droplets, which behave like bulk water, the understanding of inactivated aerosols and their activation depends fundamentally on the aerosol's physicochemical properties, which cause the socalled solute and curvature effects (Köhler, 1936). These effects enable, on the one hand, the stable existence of haze particles (also termed wetted aerosols) in subsaturated environments and inhibit, on the other hand, diffusional growth if the supersaturation does not exceed a certain threshold. This so-called critical supersaturation is associated with a critical radius, to which a wetted aerosol must grow to be considered as activated. Small aerosols activate almost immediately when the supersaturation exceeds the critical supersaturation, as it is assumed in many parameterizations of the activation process (e.g., Twomey, 1959). For larger aerosols, however, the critical radius becomes so large that the time needed for activation can be substantially increased (or prevented under certain conditions) due to the kinetically limited transport of water vapor to the particle's surface (Chuang et al., 1997). Due to their large size, however, these particles may behave like regular cloud droplets inside the environment of a cloud, although they are not formally activated (Nenes et al., 2001). Accordingly, Köhler activation theory is usually considered a weak concept for these particles. But where are the limits of Köhler activation theory located? An upper limit of the applicability of Köhler activation theory can be identified by the switch from predominantly diffusional to collectional (collision followed by coalescence) mass growth if the involved particles become large enough. Indeed, inactivated aerosols triggering collisions is closely related to the impact of giant and ultragiant aerosols (dry radius $>1 \mu \mathrm{m}$ ) on clouds, which are able to initiate precipitation due to their large wet radii 
(> $20 \mu \mathrm{m})$ (e.g., Johnson, 1982). Moreover, recent studies indicate that collection might even affect smaller particles: by considering the effects of turbulence, the collection kernel for the interaction of small particles can be significantly increased (e.g., Devenish et al., 2012). Accordingly, the main questions of this study are as follows: Where are the limits of traditional Köhler activation theory? At which aerosol size will collection dominate the activation process? And how much does collectional activation contribute to the activation of aerosols? To answer these questions, theoretical arguments and large-eddy simulations (LESs) with particle-based cloud physics are applied. Particle-based cloud physics, socalled Lagrangian cloud models (LCMs), are especially suitable for this study because they explicitly resolve the activation process and do not rely on a parameterization of it (e.g., Andrejczuk et al., 2008; Hoffmann et al., 2015; Hoffmann, 2016). Therefore, the results will give insights into the physical processes usually not covered (or missed) by those activation parameterizations typically implemented in other cloud models.

This paper is designed as follows. The following Sect. 2 will illuminate how collections can cause (or inhibit) activation by simple theoretical arguments. In Sect. 3, the LESLCM simulation setup is introduced. Results will be presented in the Sects. 4 and 5, where the former section exemplifies the applied methodology used to untangle diffusional from collectional activation and the latter section presents the results from a shallow cumulus test case. The study is summarized and discussed in Sect. 6. Appendix A introduces the governing equations of the applied LCM and necessary extensions carried out for this study.

\section{Theoretical considerations}

In this section, the general effects of coalescence on the activation of aerosols will be addressed. To simplify the argumentation in this part of the study, it is assumed that collections take place regardless of the physics that enable or inhibit them in reality. Moreover, all other microphysical processes, specifically diffusional growth, are neglected.

We consider one particle which grows by coalescing with other particles. Accordingly, the particle's water mass after $n$ collections is given by

$m_{n}=m_{0}+\sum_{i=1}^{n} m_{i}=m_{0}+n \cdot\langle m\rangle$,

where $m_{0}$ terms the particle's initial water mass and $m_{i}$ $(i>0)$ the mass of water added by each collection. The second equals sign introduces the assumption of a monodisperse ensemble of collected particles.

Based on Köhler theory, it can be shown that the critical radius for activation is given by

$r_{\text {crit }}=\sqrt{3 \frac{b \cdot m_{\mathrm{s}}}{A}}$

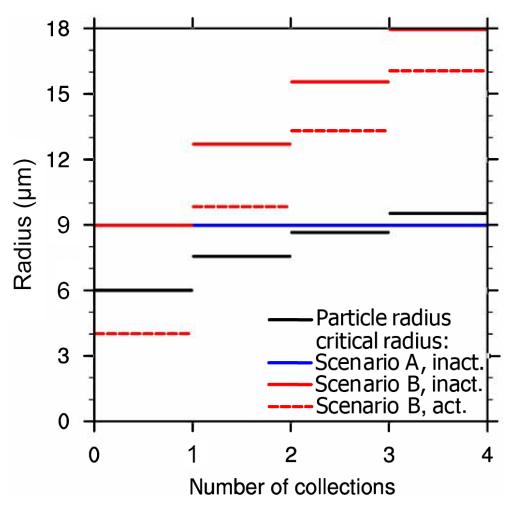

Figure 1. Change of particle radius (black line) and critical radius (colored lines) as a function of the number of collections for the growth scenarios A (negligible increase of aerosol mass, blue line) and B (aerosol mass increases proportional to the number of collections, red lines) as well as an initially inactivated (continuous lines) and an activated (dashed line) particle. The initial wet particle radius and the wet radii of the collected particles are assumed to be $6 \mu \mathrm{m}$. The initial dry aerosol mass (sodium chloride) is $2.2 \times 10^{-16} \mathrm{~kg}$ $\left(0.29 \mu \mathrm{m}\right.$ dry radius; continuous lines) and $4.4 \times 10^{-17} \mathrm{~kg}(0.17 \mu \mathrm{m}$ dry radius; dashed line). For scenario $\mathrm{B}$, the collected particles contain $2.2 \times 10^{-16} \mathrm{~kg}$ dry aerosol mass $(0.29 \mu \mathrm{m}$ dry radius $)$.

where $m_{\mathrm{S}}$ is the dry aerosol mass. Curvature effects are considered by $A=2 \sigma /\left(\rho_{1} R_{\mathrm{v}} T\right)$, depending on the surface tension of water $\sigma$, mass density of water $\rho_{1}$, specific gas constant of water vapor $R_{\mathrm{v}}$, and temperature $T$. The physicochemical aerosol properties responsible for the solute effect are represented by $b=3 v_{\mathrm{s}} \rho_{\mathrm{s}} \mu_{\mathrm{l}} /\left(4 \pi \rho_{\mathrm{l}} \mu_{\mathrm{s}}\right)$, with the van't Hoff factor $v_{\mathrm{s}}$, the mass density of the aerosol $\rho_{\mathrm{s}}$, and the molecular masses of water $\mu_{1}$ and aerosol $\mu_{\mathrm{s}}$. Accordingly, the critical mass for activation after $n$ collections yields

$m_{\text {crit }, n}=\frac{4}{3} \pi \rho_{1} \cdot r_{\text {crit }, n}^{3}=\frac{4}{3} \pi \rho_{1} \cdot\left[3 \frac{b}{A} \cdot\left(m_{\mathrm{s}, 0}+\sum_{i=1}^{n} m_{\mathrm{s}, i}\right)\right]^{3 / 2}$,

where $m_{\mathrm{s}, 0}$ terms the initial aerosol mass and $m_{\mathrm{s}, i}(i>0)$ the aerosol mass added by each collection. Approximating the summation in Eq. (3) demands further assumptions about the distribution of aerosol mass within the particle spectrum. Two scenarios are defined. In scenario A, the collected particles contain a negligible amount of aerosols. Accordingly, the aerosol mass does not change $\left(\sum_{i=1}^{n} m_{\mathrm{s}, i}=0\right)$. In scenario $\mathrm{B}$, each particle contains the same mass of aerosol. Correspondingly, the aerosol mass increases proportionally to the number of collections $\left(\sum_{i=1}^{n} m_{\mathrm{s}, i}=n \cdot\left\langle m_{\mathrm{s}}\right\rangle\right)$.

In Fig. 1, the evolving particle radius and critical radius are displayed as a function of the number of collections (details on the particle properties are given in the figure's caption). The simultaneous examination of particle radius and critical radius reveals if a particle is activated (particle radius larger than critical radius) or deactivated (particle radius smaller than critical radius). In scenario A, the initially inactivated particle (black line) grows faster than the critical 
radius (blue line), and the aerosol activates after three collections. In scenario B, an initially inactivated particle and an initially activated particle are examined (the critical radii are displayed in red by a continuous or dashed line). Since the critical radius for activation increases faster than the particle radius, activation is inhibited or the deactivation of the previously activated particle is caused.

These considerations suggest that only the collection of particles with a large amount of water and a comparably small amount of aerosol mass (i.e., highly dilute solution droplets) might lead to activation (as shown in scenario A). This, however, indicates that the collected particles are probably activated already. Therefore, the process of collectional activation will not increase the total number of activated aerosols, since one or more already activated aerosols need to be collected (and hence annihilated) in the process of one collectional activation. By contrast, the collection of particles with a comparably large amount of aerosol (i.e., less dilute solutions, as shown in scenario B) might inhibit activation since the increase of the critical radius exceeds the increase of the wet radius.

The following part of the study is investigating how coalescence is able to cause aerosol activation in shallow cumulus clouds using a detailed cloud model considering diffusional growth as well as detailed physics of collision and coalescence.

\section{Simulation setup}

The following results are derived from LES simulations applying an LCM for representing cloud microphysics. The LCM is based on a recently developed approach which simulates individual particles that represent an ensemble of identical particles and maintains, as an inherent part of this approach, the identity of droplets and their aerosols throughout the simulation (Andrejczuk et al., 2008; Shima et al., 2009; Sölch and Kärcher, 2010; Riechelmann et al., 2012; Naumann and Seifert, 2015). A summary of the governing equations and the extensions carried out for this study to treat aerosol mass change during collision and coalescence is given in the Appendix A. The underlying dynamics model, the LES model PALM (Maronga et al., 2015), solves the nonhydrostatic incompressible Boussinesq-approximated Navier-Stokes equations and prognostic equations for water vapor mixing ratio, potential temperature, and subgrid-scale turbulence kinetic energy. For scalars, a monotonic advection scheme (Chlond, 1994) is applied to avoid spurious oscillations at the cloud edge (e.g., Grabowski and Smolarkiewicz, 1990).

The initial profiles and other forcings of the simulation follow the shallow trade wind cumuli intercomparison case by Siebesma et al. (2003), which itself is based on the measurement campaign BOMEX (Holland and Rasmusson, 1973). A cyclic model domain of $3.2 \times 3.2 \times 3.2 \mathrm{~km}^{3}$ is simulated.
(In comparison to Siebesma et al. (2003), the horizontal extent has been halved in each direction due to limited computational resources.) The grid spacing is $20 \mathrm{~m}$ isotropically. Depending on the prescribed aerosol concentration, a constant time step of $\Delta t=0.2-0.5 \mathrm{~s}$ had to be used for the correct representation of condensation and evaporation, but it is also applied to all other processes. The first $1.5 \mathrm{~h}$ of simulated time are regarded as model spin-up; only the following $4 \mathrm{~h}$ are analyzed.

The simulated particles, called super-droplets following the terminology of Shima et al. (2009), are released at the beginning of the simulation, and are randomly distributed within the model domain up to a height of $2800 \mathrm{~m}$. The average distance between the super-droplets is $4.3 \mathrm{~m}$, yielding a total number of about $360 \times 10^{6}$ simulated particles and about 100 super-droplets per grid box. Initial weighting factors, i.e., the number of real particles represented by each super-droplet, are $8 \times 10^{9}, 48 \times 10^{9}, 160 \times 10^{9}, 320 \times 10^{9}$, and $640 \times 10^{9}$ for each particle, representing aerosol concentrations of $100,600,2000,4000$, and $8000 \mathrm{~cm}^{-3}$, respectively. These result in average droplet concentrations of about 48 , $220,550,750$, and $1000 \mathrm{~cm}^{-3}$, respectively.

The dry aerosol radius is assigned to each super-droplet using a random generator which obeys a typical maritime aerosol distribution represented by the sum of three lognormal distributions (Jaenicke, 1993) (Fig. 2). However, only aerosols larger than $0.005 \mu \mathrm{m}$ are initialized, since smaller aerosols do not activate in the current setup. The different aerosol concentrations are created by scaling the weighting factor of each simulated particle to attain the desired concentration. The aerosols are assumed to consist of sodium chloride $\left(\mathrm{NaCl}\right.$, mass density $\rho_{\mathrm{s}}=2165 \mathrm{~kg} \mathrm{~m}^{-3}$, van't Hoff factor $v_{\mathrm{s}}=2$, molecular weight $\mu_{\mathrm{s}}=58.44 \mathrm{~g} \mathrm{~mol}^{-1}$ ). The initial wet radius of each super-droplet is set to its approximate equilibrium radius depending on aerosol mass and ambient supersaturation (Eq. 14 in Khvorostyanov and Curry, 2007). The applied collection kernel includes effects of turbulence, which have been shown to increase the collection probability of small particles significantly (e.g., Devenish et al., 2012). See Appendix A for more details on the applied LCM.

\section{Methodology}

In this section, the applied methodology for untangling the contributions of diffusion and collection to the activation of aerosols is introduced. An aerosol becomes activated when it grows beyond its critical radius ( $\left.r>r_{\text {crit }}\right)$. Moreover, activation requires the particle to be located in a volume of air with sufficient supersaturation to enable unhindered diffusional growth. Depending on the microphysical process responsible for the final crossing of $r_{\text {crit }}$, different supersaturation allow unhindered diffusional growth. 


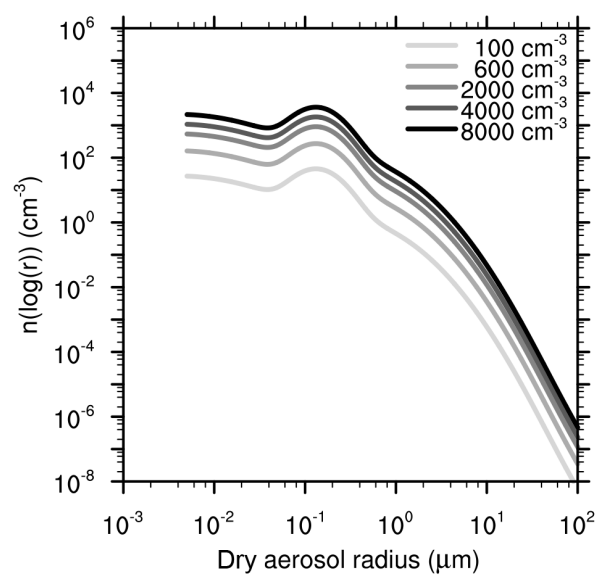

Figure 2. The number density distribution of dry aerosol radii for different aerosol concentrations (line brightness).

The supersaturation has to be larger than the critical supersaturation in the moment in which the critical radius is exceeded:

$S>S_{\text {crit }}=S_{\text {eq }}\left(r_{\text {crit }}\right)$,

where $S_{\text {eq }}$ is the equilibrium supersaturation calculated according to Köhler theory (see Eq. A3). This condition is automatically fulfilled in the case of diffusional growth due to the constraints of Köhler theory on the equilibrium supersaturation. If the critical radius is exceeded by collection, the radius after collection might be immediately larger than $r_{\text {crit }}$ and, hence, the necessary supersaturation is allowed to be smaller to enable unhindered diffusional growth:

$S \geq S_{\text {eq }}\left(r_{\mathrm{ac}}\right)$,

where $r_{\mathrm{ac}} \geq r_{\text {crit }}$ is the wet radius after collection. This criterion is not automatically fulfilled and is checked additionally to establish the formal equivalence of both processes, i.e., enabling unhindered diffusional growth after activation. Note that the process of activation, i.e., the entire growth beyond $r_{\text {crit }}$, can be driven by diffusional growth or by accumulating liquid water due to collection or by a combination of both.

To decide whether an activation is primarily driven by diffusion or collection, all simulated particles have been tracked throughout the simulation and their mass growth has been integrated from their minimum mass before activation, $\min (m)$, to the critical activation mass, $m_{\text {crit }}$ :

$$
\begin{aligned}
& \left.\Delta m\right|_{\text {diff }}=\left.\int_{\min (m)}^{m_{\text {crit }}} \mathrm{d} m\right|_{\text {diff }}, \\
& \left.\Delta m\right|_{\text {coll }}=\left.\int_{\min (m)}^{m_{\text {crit }}} \mathrm{d} m\right|_{\text {coll }},
\end{aligned}
$$

where $\left.\mathrm{d} m\right|_{\text {diff }}$ and $\left.\mathrm{d} m\right|_{\text {coll }}$ are directly derived from the LCM model Eqs. (A2) and (A6)-(A7), respectively. Note the fol- (i)

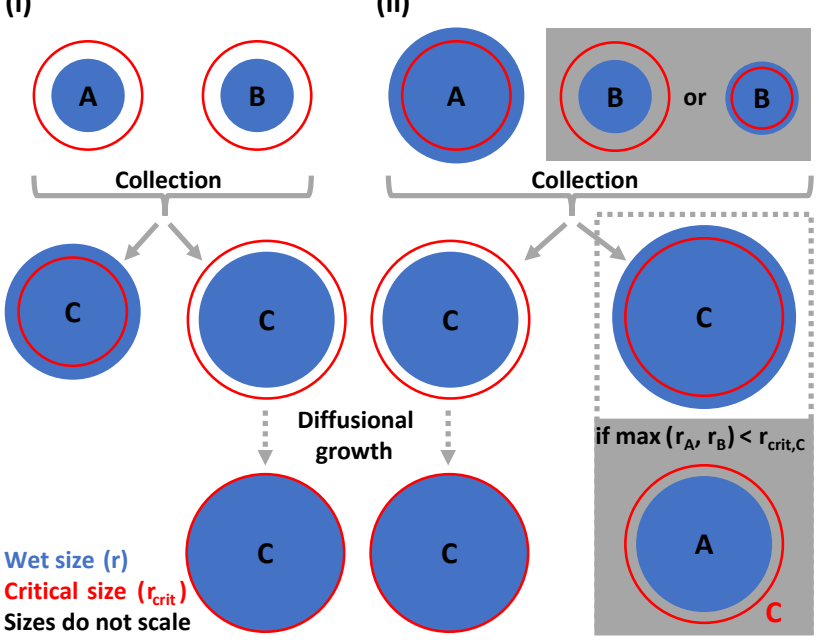

Figure 3. Possible microphysical processes leading to the collectional activation of particle C. Scenario (i) contains only inactivated aerosols, scenario (ii) contains at least one activated aerosol. The blue circle displays the wet size of the particle, the red circle the critical size, which has to be exceeded for activation. The displayed sizes do not scale.

lowing procedures for determining $\min (m),\left.\Delta m\right|_{\text {diff }}$, and $\left.\Delta m\right|_{\text {coll }}$ during the simulation: (i) if a particle shrinks below $\min (m)$ before activation, $\left.\Delta m\right|_{\text {diff }}$ and $\left.\Delta m\right|_{\text {coll }}$ are set to zero and are re-calculated starting from this new minimum mass; (ii) if a particle becomes deactivated, i.e., evaporates and becomes smaller than its critical radius after being activated, the current mass is considered the new $\min (m)$, and $\left.\Delta m\right|_{\text {diff }}$ and $\left.\Delta m\right|_{\text {coll }}$ are set to zero; (iii) if a collection does not result in an activation and the particle evaporates back to its equilibrium radius afterwards, $\left.\Delta m\right|_{\text {diff }}$ will be negative and $\left.\Delta m\right|_{\text {coll }}$ positive. To avoid the potentially incorrect classification of a following activation, $\left.\Delta m\right|_{\text {diff }}$ and $\left.\Delta m\right|_{\text {coll }}$ are set to zero if $\left.\Delta m\right|_{\text {diff }}$ becomes negative, and the current mass is considered as $\min (m)$.

To identify a collectional activation, the integrated collectional mass growth $\left.\Delta m\right|_{\text {coll }}$ is compared to the diffusional $\left.\Delta m\right|_{\text {diff }}$ in the moment the particle grows beyond its critical radius. If the former exceeds the latter $\left(\left.\Delta m\right|_{\text {coll }}>\left.\Delta m\right|_{\text {diff }}\right)$, this activation is considered as collectional. There are various microphysical interactions resulting in $\left.\Delta m\right|_{\text {coll }}>\left.\Delta m\right|_{\text {diff }}$, and its basic types are illustrated in Fig. 3. Note also that a combination or a repetition of these types is possible, i.e., multiple subsequent collections. In a collectional activation of type (i), the water mass growth by collection dominates, i.e., the coalescence of two previously inactivated aerosols $\mathrm{A}$ and $\mathrm{B}$ results directly or after some diffusional growth in an activated particle C. In collectional activations of type (ii), the critical radius increases faster than wet radius, i.e., the coalescence of an already activated particle A with another activated or an inactivated particle B results in inactivated particle $\mathrm{C}$, which activates after some diffusional growth. If 

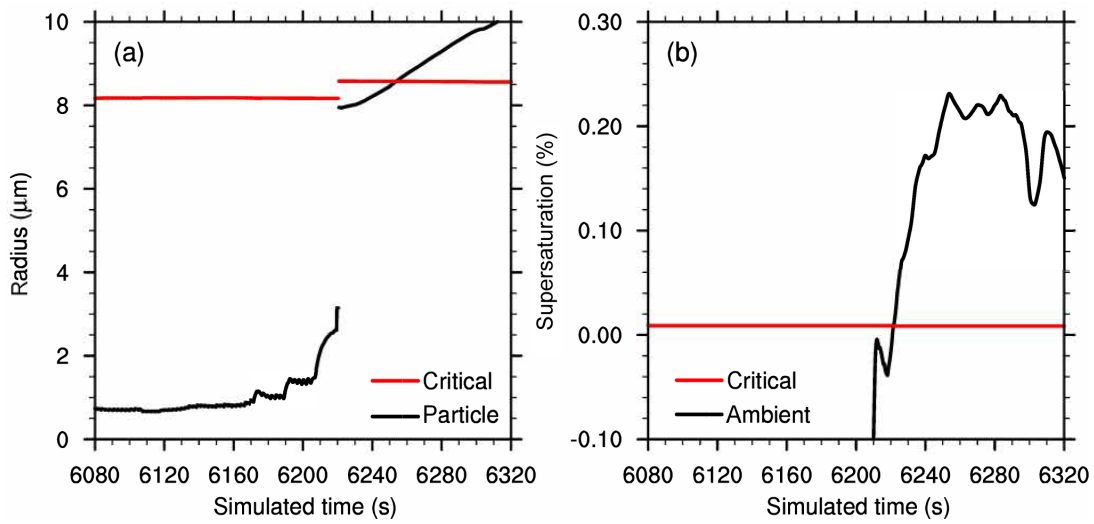

Figure 4. Time series of a particle which is activated by collection. Panel (a) shows its radius (black) and critical radius (red), and panel (b) depicts the ambient supersaturation experienced by that particle (black) and its critical supersaturation (red).

the resulting particle is directly activated, this process is only considered a collectional activation if the largest wet radius of the two coalescing particles A and B is smaller than the critical radius of the newly produced particle $\mathrm{C}$ :

$\max \left(r_{\mathrm{A}}, r_{\mathrm{B}}\right)<r_{\text {crit, } \mathrm{C}}$

This ensures that the combined water of particles A and B is necessary to activate particle $\mathrm{C}$. If this is not the case, i.e., the water of particle $\mathrm{A}$ or $\mathrm{B}$ is able to activate particle $\mathrm{C}$ on its own, the latter process is considered a regular collection of cloud droplets or as scavenging and neglected in the following analysis. Moreover, the coalescence of two activated particles resulting in a collectional activation is mathematically possible but not found to play a role in the analyzed simulations. Note that only collectional activations of the first type are able to increase the number of activated aerosols, while the second type might have no impact or a negative impact on the total number of activated aerosols since the coalescence of at least one activated particle results in one activated particle.

To exemplify this methodology, Fig. 4 shows, for an aerosol selected from the LCM simulations discussed below, the time series of its radius and critical radius (panel (a)) and the ambient supersaturation and critical supersaturation (panel (b)). Note that this aerosol is actually one superdroplet, representing a larger ensemble of identical aerosols, which is, however, interpreted as one aerosol here. The initial dry radius of the aerosol is $0.27 \mu \mathrm{m}$. On its way to activation, the particle experiences diffusional growth, which can be easily identified by the continuous change of radius. One collection event, characterized by a distinct increase in radius, is visible at $6220 \mathrm{~s}$ simulated time. At this point in time, the inactivated aerosol (wet radius $3.1 \mu \mathrm{m}$ ) coalesces with an activated particle (wet radius $7.8 \mu \mathrm{m}$, aerosol dry radius $0.13 \mu \mathrm{m}$ ), but the product of coalescence (wet radius $7.9 \mu \mathrm{m}$, aerosol dry radius $0.28 \mu \mathrm{m}$ ) remains inactivated. Due to the increased amount of aerosol mass, the critical radius (and to a lesser extent the critical supersaturation) increases (decreases) after the coalescence. Afterwards, the particle grows by diffusion and exceeds the critical radius at $6253 \mathrm{~s}$ simulated time, which can be identified as the time of activation. All in all, this activation is considered a collectional activation since $\left.\Delta m\right|_{\text {coll }}=1.9 \times 10^{-12} \mathrm{~kg}>\left.\Delta m\right|_{\text {diff }}=$ $6.2 \times 10^{-13} \mathrm{~kg}$. Moreover, this is a collectional activation of type (ii) since it involves the collection of an already activated aerosol.

\section{Results}

The last section showed that collection can contribute significantly to the mass growth leading to the activation of a single aerosol. But how does collection contribute to the activation of aerosols in general? Figure 5 shows the vertical profiles of (a) the maximum diffusion radius, i.e., the largest critical radius of an aerosol activated exclusively by diffusion at a certain height; (b) the supersaturation; (c) the collectional activation rate, i.e., the number of aerosols activated by collection per unit volume and unit time; and (d) the corresponding diffusional activation rate. Profiles (b) to (d) are conditionally averaged over all supersaturated grid cells. Only data of the last 4 simulated hours are considered. Values above the average cloud-top height (at $1500 \mathrm{~m}$ ) are not displayed due to insufficient statistics.

The maximum diffusion radius (Fig. 5a) increases almost monotonically with height, reaching maxima between 30 and $9 \mu \mathrm{m}$ for aerosol concentrations of 100 to $8000 \mathrm{~cm}^{-3}$, respectively. The supersaturation (Fig. 5b) exhibits a distinct peak at the cloud base and relaxes toward its equilibrium value, which is determined by the number of activated aerosols and vertical velocity above (e.g., Rogers and Yau, 1989, Chap. 7). Due to the larger number of water vapor absorbers, the supersaturation and the maximum diffusion radius are generally smaller in the more aerosol-laden simulations. 

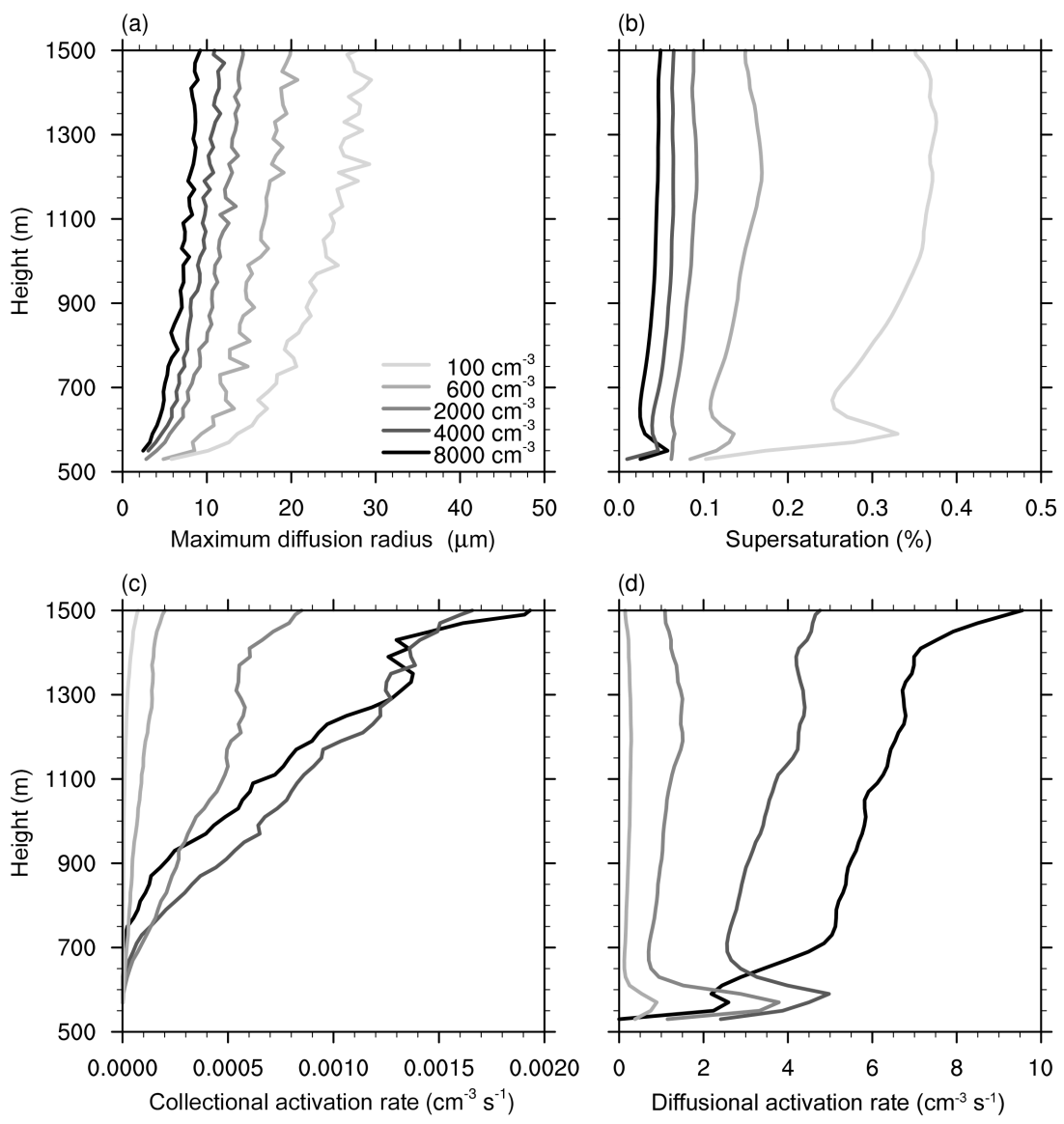

Figure 5. Vertical profiles of the maximum diffusion radius (a), the supersaturation (b), the collectional activation rate (c), and the diffusional activation rate (d) for the analyzed aerosol concentrations (line brightness).

The collectional activation rate (Fig. 5c) increases almost linearly with height. This increase can be related to the longer-lasting diffusional growth, resulting in potentially larger particles at higher levels, which increases the collection kernel and therefore the collection probability. The slope is larger in aerosol-laden environments, where more aerosols are available for activation. Additionally, the height above cloud base, where the collectional activation starts, increases with the aerosol concentration since the average particle radius is too small to enable collisions at lower levels. Accordingly, the collectional activation rate in the $8000 \mathrm{~cm}^{-3}$ simulation exhibits smaller to similar values than in the $4000 \mathrm{~cm}^{-3}$ simulation, although the slope in the $8000 \mathrm{~cm}^{-3}$ simulation is larger. The shape of the collectional activation rate differs significantly from the typical profile of the diffusional activation rate (Fig. 5d), which appears as a distinct peak at cloud base where the majority of aerosols activate after the entrainment through the cloud base in clean conditions (Slawinska et al., 2012; Hoffmann et al., 2015). In more aerosol-laden conditions, a larger fraction of diffusional activations occurs at higher levels. In these simulations, only larger aerosols are able to activate by diffu- sion due to the generally lower supersaturations. These larger aerosols, however, need a longer time to activate. Accordingly, these aerosols are lifted to higher levels by the cloud's updraft until they grow beyond their critical radius for activation with commensurate changes in the profile of the diffusional activation rate.

The comparison of the numerical values of the activation rates in Fig. 5c and d indicate already that the contribution of collectional activation to the number of activated aerosols is significantly smaller than the contribution of diffusional activation. Figure 6 shows that only 1 activation in 10000 to 35000 is caused by collection, with a greater contribution of collectional activation in moderately aerosol-laden environments up to $4000 \mathrm{~cm}^{-3}$. As it will be outlined below, this increase can be attributed to a shift of collectional activation to smaller, but more numerous aerosols. For $8000 \mathrm{~cm}^{-3}$, however, the fraction decreases again since the particles are too small to trigger a larger amount of collisions.

Figure 7 shows the collectional and diffusional fraction of activations as a function of the dry aerosol radius on the lower abscissa and the corresponding critical radius (calculated for the cloud-base temperature of approximately $294.5 \mathrm{~K}$ ) on 


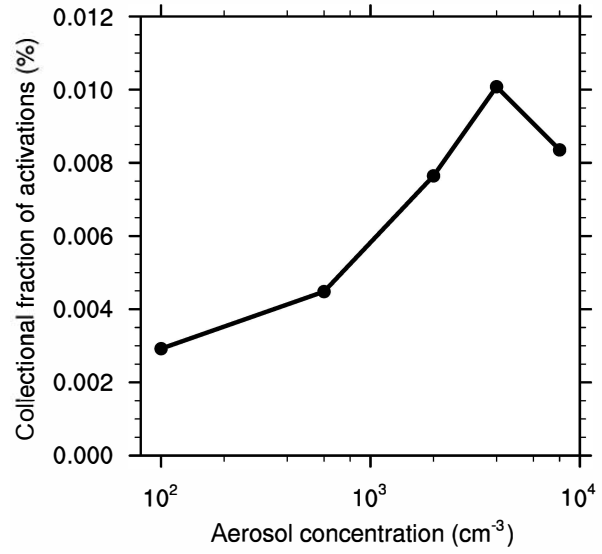

Figure 6. The collectional fraction of all activations as a function of the aerosol concentration.

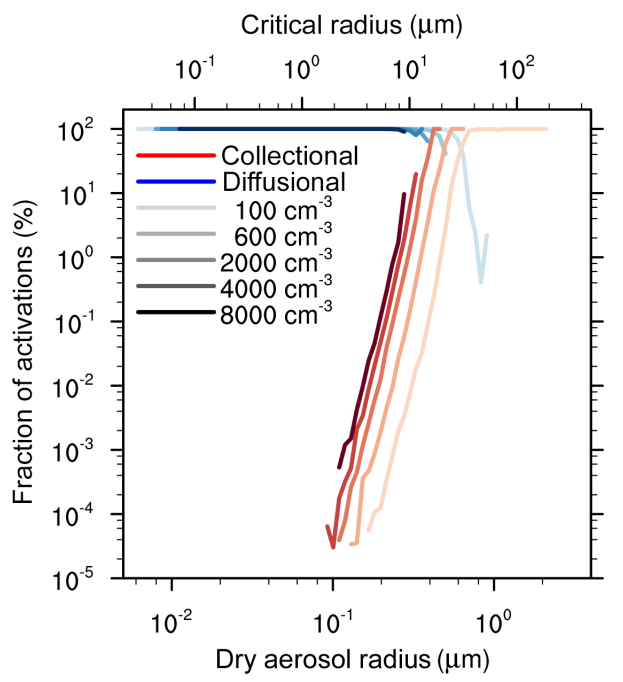

Figure 7. The collectional (red lines) and diffusional (blue lines) fraction of activations as a function of the dry aerosol radius (lower abscissa) and critical radius (at cloud-base temperature of $294.5 \mathrm{~K}$, upper abscissa) for the analyzed aerosol concentrations (line brightness).

the upper abscissa. As expected, diffusional activation is the dominant process for small aerosols (dry radius $<0.1 \mu \mathrm{m}$ ) as long as the dry aerosol radius is not too small and the corresponding critical supersaturation not too high to inhibit activation. Accordingly, the left boundary of diffusional activation is shifted toward larger radii as the maximum supersaturations decrease in more aerosol-laden environments (see Fig. 5b). For aerosols larger than $0.1 \mu \mathrm{m}$, collectional activation becomes increasingly important, affecting aerosols in the range of $0.16-2.5,0.13-0.65,0.11-0.46,0.092-0.33$, and $0.11-0.28 \mu \mathrm{m}$ for aerosol concentrations of 100,600 , 2000,4000 , and $8000 \mathrm{~cm}^{-3}$, respectively. Larger aerosols do not activate at all, since their critical radius is too large to be exceeded by diffusion or collection.

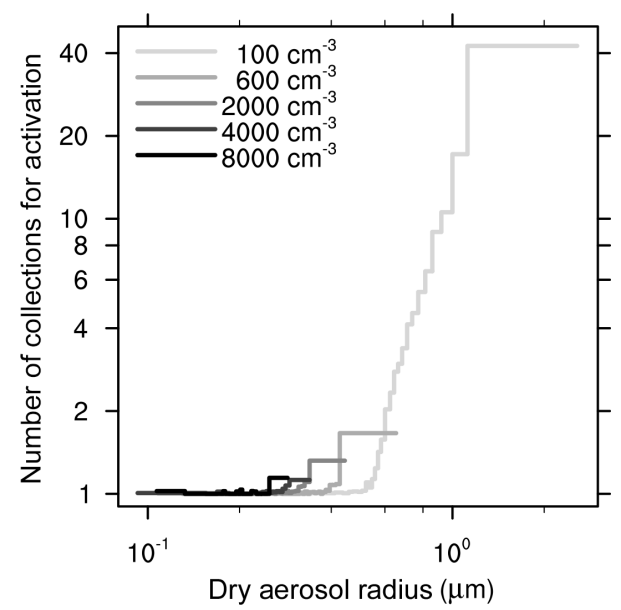

Figure 8. The average number of collections necessary to cause a collectional activation as a function of the dry aerosol radius for the analyzed aerosol concentrations (line brightness). The data have been binned; each bin contains at least $3 \%$ of all registered collectional activations.

The collectional fraction of activations increases following a power-law relation toward larger radii, reflecting the higher collision probability of larger particles. The collectional fraction reaches up to $100 \%$ for the 100,600 , and $2000 \mathrm{~cm}^{-3}$ simulations at about $0.83,0.54$, and $0.42 \mu \mathrm{m}$ dry aerosol radius, respectively, indicating a significant effect of collectional activation on this part of the aerosol spectrum. For higher aerosol concentrations, collectional activation does not dominate but still contributes with fractions up to 20 and $10 \%$ for aerosol concentrations of 4000 and $8000 \mathrm{~cm}^{-3}$, respectively. The dry aerosol radius at which collectional activation reaches $100 \%$ matches the maximum radii that can be produced by diffusion. To create any larger particles, existing particles need to be merged. Accordingly, to activate aerosols with a larger critical radius, collection must be inherently involved. For the $100 \mathrm{~cm}^{-3}$ simulation, the largest radii produced by diffusion are about $30 \mu \mathrm{m}$ (Fig. 5a), corresponding to a dry aerosol radius of $0.63 \mu \mathrm{m}$, which is close to the first dry aerosol radii exhibiting a $100 \%$ collectional fraction of activations. A similar agreement can be found for the simulations initialized with aerosol concentrations of 600 and $2000 \mathrm{~cm}^{-3}$.

In general, the range of aerosols affected by collectional activation shifts toward smaller radii as the aerosols concentration increases. This is primarily a result of the decreasing maximum radii that can be reached by diffusion alone (Fig. 5a). Additionally, the supersaturation decreases too (Fig. 5b), which decelerates diffusional activation and therefore favors collectional activation. Since small aerosols are significantly more abundant than larger ones (Fig. 2), the number of aerosols that are potentially activated by collection increases as a result of this shift, resulting in the larger collectional fraction of all activations shown in Fig. 6 . 
How many collections are necessary for the collectional activation of one aerosol? Figure 8 displays the average number of collisions that take place during a collectional activation. For dry aerosol radii up to $0.3-0.5 \mu \mathrm{m}$ (depending on aerosol concentration), only one collection is necessary to cause activation. For larger aerosols more collections are needed: up to 42 collections for the activation of aerosols with a dry radius of more than $1.0 \mu \mathrm{m}$. As illustrated in Fig. 3, not all of these collections involve the coalescence of inactivated aerosols, which would result in an increase in the number of activated aerosols. In fact, some collections involve already activated aerosols, which results in a neutral or negative impact of collectional activation on the total number of activated particles. To quantify the influence on the number of activated aerosols, the effective activation ratio is defined: the net increase in the number of newly activated aerosols per collectional activation. Figure 9 displays the effective activation ratio calculated from all registered collectional activations. For an aerosol concentration of $100 \mathrm{~cm}^{-3}$, where a large portion of aerosols needs multiple collections for activations (Fig. 8), the effective activation ratio is -1.2 , i.e., more activated aerosols are annihilated than produced to enable the final activation of one aerosol by collection. But for an aerosol concentration of $600 \mathrm{~cm}^{-3}$ and more, the effective activation ratio becomes positive and is approximately constant at 0.4 , indicating that on average 0.4 new activated aerosols are produced per collectional activation. This ratio has to be considered in the interpretation of the collectional fraction of all activations (Fig. 6), indicating that the net effect of collectional activation is actually smaller (or even negative).

Although activation is dominated by collectional mass growth for larger aerosols, the growth by diffusion is still essential to create sufficiently large particles to trigger collisions. Figure 10a depicts the collectional fraction of mass growth needed to grow beyond the critical mass for activation (for aerosols activated by collection). Note that the diffusional fraction of mass growth is the remaining fraction. For the smallest affected aerosols $(\sim 0.1 \mu \mathrm{m})$, the collectional fraction of mass growth is about $75 \%$ and decreases slightly to $65 \%$ for aerosols of $\sim 0.4 \mu \mathrm{m}$, indicating that a large contribution of diffusional growth is necessary to produce sufficiently large particles that are able to collide. The slight increase toward smaller radii indicates that collectional activation is only possible for the smallest aerosols if they encounter a substantially larger particle. For aerosols larger than $1 \mu \mathrm{m}$, the collectional fraction increases rapidly to $97 \%$, which can be attributed to the large critical radii which can be only exceeded by the collection of multiple droplets (cf. Fig. 8).

Figure 10b displays the mean entrainment height of the particles involved in each collectional activation. Apart from the largest particles $(>0.6 \mu \mathrm{m})$ in the most pristine case $\left(100 \mathrm{~cm}^{-3}\right)$, all collectional activations involve particles that have entered the cloud well above the cloud base, which is

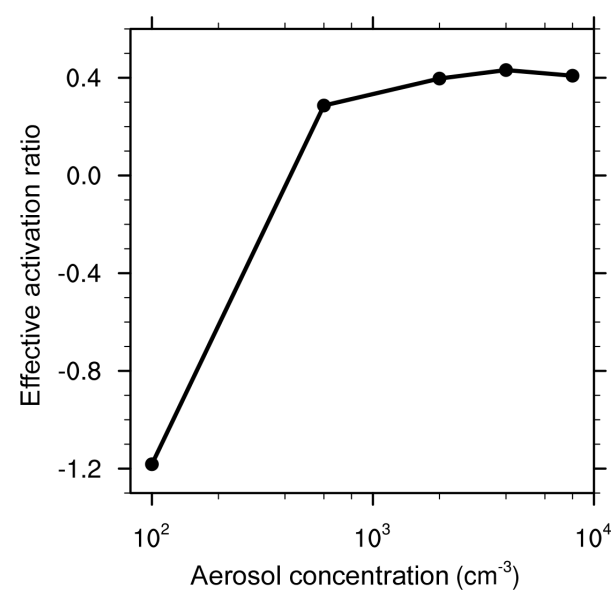

Figure 9. The effective activation ratio (i.e., the net increase in the number of newly activated aerosols per collectional activation) as a function of aerosol concentration.

located at 500-600 m. Accordingly, these particles miss the typical supersaturation maximum located at cloud base (see Fig. 5b), where the majority of aerosols activate by diffusion. Indeed, entrainment above cloud base is generally favorable for collectional activation, since these aerosols are mixed into an environment where larger particles exist, triggering collisions among them more easily. For aerosols larger than $0.6 \mu \mathrm{m}$, the average entrainment height is located closer to the cloud base. Since multiple collections are necessary for their activation (see Fig. 8), the lower average entrainment height is representative for the average entrainment height of all particles inside the cloud, which is the cloud base through which most particles enter the cloud (e.g., Hoffmann et al., 2015).

\section{Summary and discussion}

The influence of collision and coalescence on the activation of aerosols has been studied using theoretical arguments and large-eddy simulations with a coupled Lagrangian cloud model. The presented theory has shown that an inactivated aerosol can be activated by the collection of particles with a comparably small amount of aerosol mass (i.e., particles consisting almost entirely of water), while the collection of large amounts of additional aerosol mass inhibits activation or even causes the deactivation of previously activated aerosols. The LCM simulations of shallow trade wind cumuli indicated that collectional activation becomes possible for aerosols larger than approximately $0.1 \mu \mathrm{m}$ in dry radius, and its contribution increases with a power-law relation toward larger aerosols. In pristine conditions, collection is the only process for the activation of aerosols larger than $0.83 \mu \mathrm{m}$ in dry radius at an aerosol concentration of $100 \mathrm{~cm}^{-3}$. This boundary is shifted to smaller radii in more polluted environments (down to $0.42 \mu \mathrm{m}$ at $2000 \mathrm{~cm}^{-3}$ ). The highest contribu- 

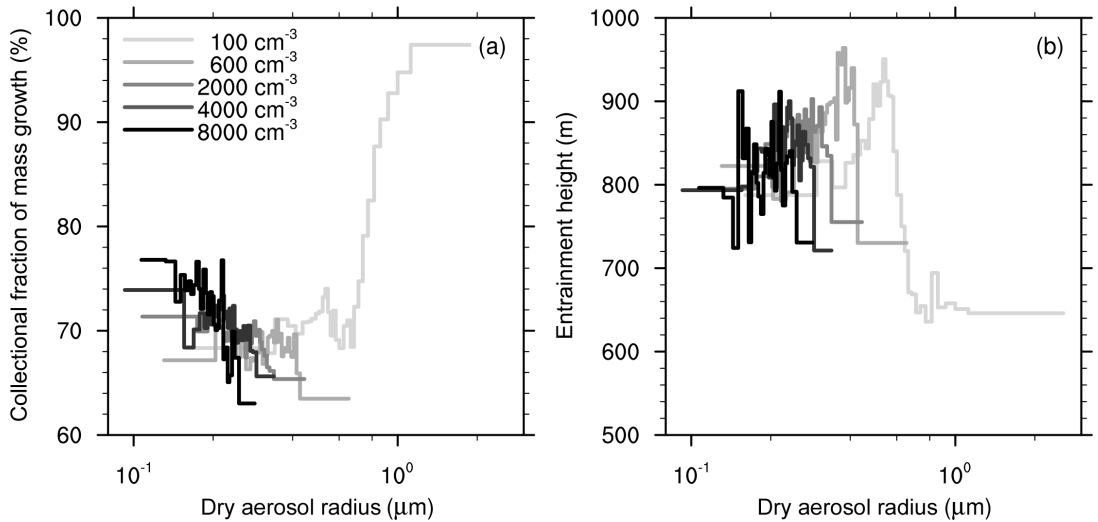

Figure 10. Collectional fraction of (a) the mass growth leading to collectional activation, and (b) the average entrainment height as a function of the dry aerosol radius for the analyzed aerosol concentrations (line brightness). The data have been binned; each bin contains at least $3 \%$ of all registered collectional activations.

tion of collectional activation to the total number of activated aerosols is found at an aerosol concentration of $4000 \mathrm{~cm}^{-3}$, where 1 in 10000 activations is caused by collection. If the aerosol concentration becomes higher and hence the particles too small, collectional activation is inhibited and its contribution decreases again. Collectional activation frequently involves the collection of already activated aerosols reducing the net increase of newly activated aerosols per collectional activation to 0.4 , while the remainder ( 0.6 activated aerosols) is annihilated during the activation process. Moreover, collectional activation predominantly affects particles that have been entrained above cloud base, i.e., above the region of the cloud where the highest supersaturations occur. Accordingly, these particles experience systematically lower supersaturations, which prevents diffusional activation. Finally, it has been shown that the collectional activation rate increases almost linearly with height, while the slope and the height, from which collectional activation starts, increase with the aerosol concentration.

In conclusion, this study revealed collision and coalescence as an additional process for the activation of aerosols. This process is not covered by commonly applied activation parameterizations (e.g., Twomey, 1959). But does this matter? First of all, with a maximum of 1 in 10000 activations, collectional activation can be safely neglected. But one can also argue that collectional activation is already (but implicitly) covered by standard cloud models: activation parameterizations usually activate aerosols as soon as the critical supersaturation is exceeded, i.e., they neglect kinetic effects inhibiting the immediate activation of large aerosols, which need a certain time to grow beyond their critical radius. As pointed out by Chuang et al. (1997), this might overestimate the number of activated aerosols (or cloud droplets) since a certain fraction of the larger aerosols is falsely treated as activated. Following the argumentation of Nenes et al. (2001), these particles might act, however, as regular cloud droplets due to their large wet radii despite not being formally ac- tivated, and the estimated droplet number concentration is a valid measure for particles that behave like cloud droplets. And indeed, this study showed that a certain fraction of these formally inactivated particles are able to collide and coalesce, i.e., act as regular cloud droplets. Similarly, in standard cloud models, these falsely activated cloud droplets will experience the model's representation of collision and coalescence that might ultimately result in an implicit realization of collectional activation.

Accordingly, collectional activation is not of particular importance for determining the number of cloud droplets, but it indicates clearly the limits of Köhler activation theory. Without ambiguity, Köhler activation theory is only applicable to aerosols smaller than $0.1 \mu \mathrm{m}$ in dry radius, while an increasing fraction of aerosols activates by collection at larger radii. Ultimately, the activation of aerosols larger than about $1.0 \mu \mathrm{m}$ is entirely caused by collection (if it takes place at all). Therefore, the range between approximately 0.1 and $1.0 \mu \mathrm{m}$ should be considered as a transition zone between (i) typical aerosols that need to experience sufficiently strong supersaturations to grow beyond the critical radius and (ii) so-called giant and ultragiant aerosols with sufficiently large wet radii to act like cloud droplets by triggering collision and coalescence without being formally activated (e.g., Johnson, 1982).

Finally, potential sources of uncertainty within this study shall be mentioned. First, the accuracy of the applied collection kernel is limited. The widely used collision efficiencies of Hall $(1980)$ for small particles $(\lesssim 20 \mu \mathrm{m})$ are slightly higher than other estimates (e.g., Böhm, 1992). An effect of this uncertainty might be the collectional activation of aerosols that are too small to collide in reality. Moreover, the collection kernel might not incorporate all processes relevant for collections among aerosols and droplets. For instance, Brownian diffusion might increase the collection of smaller particles (e.g., Ardon-Dryer et al., 2015) but might not lead to collectional activation since it will predominantly add aerosol mass and only a small amount of water 
(cf. Sect. 2). Additional simulations neglecting turbulence effects on the collection kernel (not shown) have exhibited a similar spectral distribution of collectional activation, but indicated a smaller contribution to the total number of activated aerosols. Additionally, the collection algorithm itself might underestimate collisions due to the initial distribution of weighting factors (Unterstrasser et al., 2017), and the determined influence of collectional activation should be considered as a lower estimate. Second, the initialized aerosol distribution is always maritime, i.e., it includes a large fraction of large aerosols which are not part of continental air masses (e.g., Jaenicke, 1993) but are primarily affected by collectional activation as shown here. Accordingly, the collectional fraction of activations might be lower in environments which exhibit a smaller fraction of aerosols in the affected size range. Third, not all aerosols consist of (highly hygroscopic) sodium chloride, although the size range affected by collectional activation is usually assumed to consist of sea salt (Jaenicke, 1993). Aerosols with a lower hygroscopicity would exhibit a smaller solution effect which is equivalent to a smaller dry radius of the sodium chloride aerosols examined here, i.e., the wet radius of these aerosols would be smaller and they would be less likely to cause collisions. Again, the range of aerosols affected by collectional activation would be shifted to larger dry radii.

Code availability. The applied LES-LCM model is freely available (revision 1954, http://palm.muk.uni-hannover.de/trac/browser/ ?rev=1954). Additional software developed for the LES-LCM model as well as the analysis is available on request. 


\section{Appendix A: The Lagrangian cloud model}

In this section, the basic framework of the Lagrangian cloud model (LCM) applied in this study, as well as the extensions made to treat aerosol mass during collision and coalescence, is described. One can refer to Riechelmann et al. (2012) for the original description, Hoffmann et al. (2015) for the consideration of aerosols during diffusional growth, and Hoffmann et al. (2017) for the most recent description of the LCM. This LCM, as with all other available particle-based cloud physical models (Andrejczuk et al., 2008; Shima et al., 2009; Sölch and Kärcher, 2010; Naumann and Seifert, 2015), is based on the so-called super-droplet approach in which each simulated particle represents an ensemble of identical, real particles, growing continuously from an aerosol to a cloud droplet. The number of particles within this ensemble, the so-called weighting factor, is a unique feature of each particle, which is considered for an appropriate physical representation of cloud microphysics within the super-droplet approach.

The transport of a simulated particle is described by

$$
\frac{\mathrm{d} X_{i}}{\mathrm{~d} t}=u_{i}+\tilde{u_{i}}-\delta_{i 3} w_{\mathrm{s}},
$$

where $X_{i}$ is the particle location and $u_{i}$ is the LES resolvedscale velocity at the particle location, determined from interpolating linearly between the eight adjacent grid points of the LES. A turbulent velocity component $\widetilde{u_{i}}$ is computed from a stochastic model based on the LES subgrid-scale turbulence kinetic energy (Sölch and Kärcher, 2010). The sedimentation velocity $w_{\mathrm{s}}$ is given by an empirical relationship (Rogers et al., 1993). Equation (A1) is solved using a firstorder Euler method.

As described in Hoffmann et al. (2015), the diffusional growth of each simulated particle is calculated from

$r \frac{\mathrm{d} r}{\mathrm{~d} t}=\frac{S-S_{\mathrm{eq}}}{F_{\mathrm{k}}+F_{\mathrm{D}}} \cdot f\left(r, w_{\mathrm{s}}\right)$,

where $r$ is the particle's radius and $S$ terms the supersaturation within the grid box in which the particle is located. Curvature and solution effects are considered by the equilibrium supersaturation

$S_{\text {eq }}=\frac{A}{r}-\frac{b \cdot m_{\mathrm{s}}}{r^{3}}$.

The factor $f$ parameterizes the so-called ventilation effect (Rogers and Yau, 1989). The coefficients $F_{\mathrm{k}}=\left(L_{\mathrm{v}} /\left(R_{\mathrm{v}} T\right)-\right.$ 1) $\cdot L_{\mathrm{v}} \rho_{\mathrm{l}} /(T k)$ and $F_{\mathrm{D}}=\rho_{\mathrm{l}} R_{\mathrm{v}} T /\left(D_{\mathrm{v}} e_{\mathrm{s}}\right)$ represent the effects of thermal conduction and diffusion of water vapor between the particle and the surrounding air, respectively. Here, $k$ is the coefficient of thermal conductivity in air, $D_{\mathrm{v}}$ is the molecular diffusivity of water vapor in air, $L_{\mathrm{V}}$ is the latent heat of vaporization, and $e_{\mathrm{S}}$ is the saturation vapor pressure. Equation (A2) is solved using a fourth-order Rosenbrock method.
Collision and coalescence are calculated from a statistical approach in which collections are calculated from the particle size distribution resulting from all super-droplets currently located within a grid box. These interactions affect the weighting factor $A_{n}$ (i.e., the number of all particles represented by one super-droplet), the total water mass of a superdroplet $M_{n}=A_{n} \cdot m_{n}$ (where $m_{n}$ is the mass of one particle represented by super-droplet $n$ ), and also the dry aerosol mass $M_{\mathrm{s}, n}=A_{n} \cdot m_{\mathrm{s}, n}$ (where $m_{\mathrm{s}, n}$ is the dry aerosol mass of one particle represented by super-droplet $n$ ). The latter interaction has been introduced for this study. The algorithm follows the all-or-nothing principle (Shima et al., 2009; Sölch and Kärcher, 2010), which has been rigorously evaluated by Unterstrasser et al. (2017) and has been recently incorporated into this LCM by Hoffmann et al. (2017).

It is assumed that the super-droplet with the smaller weighting factor (index $n$ ) collects $A_{n}$ particles from the super-droplet with the larger weighting factor (index $m$ ), with commensurate changes in $M_{m}, M_{n}, M_{\mathrm{s}, m}$, and $M_{\mathrm{s}, n}$. Since the weighting factor of the collecting super-droplet $n$ does not change during this process, its wet radius

$r_{n}=\left(\frac{M_{n}}{\frac{4}{3} \pi \rho_{1} A_{n}}\right)^{1 / 3}$

and the dry aerosol radius

$r_{\mathrm{s}, n}=\left(\frac{M_{\mathrm{s}, n}}{\frac{4}{3} \pi \rho_{\mathrm{s}} A_{n}}\right)^{1 / 3}$

increase. Additionally, same-size collections of the particles belonging to the same super-droplet are considered. These interactions do not change $M_{n}$ and $M_{\mathrm{s}, n}$, but they decrease $A_{n}$ and accordingly increase $r_{n}$ and $r_{\mathrm{s}, n}$.

These two processes yield, in the following description for the temporal change of $A_{n}$ (assuming that the simulated particles are sorted such that $\left.A_{n}>A_{n+1}\right)$,

$$
\frac{\mathrm{d} A_{n}}{\mathrm{~d} t} \delta t=-\frac{1}{2}\left(A_{n}-1\right) P_{n n}-\sum_{m=n+1}^{N_{\mathrm{p}}} A_{m} P_{m n} .
$$

The first term on the right-hand side denotes the loss of $A_{n}$ due to same-size collections; the second term denotes the loss of $A_{n}$ due to collisions with particles of a smaller weighting factor. The total water mass and the total aerosol mass of a super-droplet change according to

$$
\frac{\mathrm{d} M_{n}}{\mathrm{~d} t} \delta t=\sum_{m=1}^{n-1} A_{n} m_{m} P_{n m}-\sum_{m=n+1}^{N_{\mathrm{p}}} A_{m} m_{n} P_{m n}
$$

and

$$
\frac{\mathrm{d} M_{\mathrm{s}, n}}{\mathrm{~d} t} \delta t=\sum_{m=1}^{n-1} A_{n} m_{\mathrm{s}, m} P_{n m}-\sum_{m=n+1}^{N_{\mathrm{p}}} A_{m} m_{\mathrm{s}, n} P_{m n},
$$


respectively. In both equations, the first term on the righthand side denotes the increase of $M_{n}$ or $M_{\mathrm{s}, n}$ by the collection of water or dry aerosol mass from super-droplets with a larger weighting factor, while the second term describes the loss of these quantities to super-droplets with a smaller weighting factor. The function $P_{m n}$ determines whether a collection takes place:

$P_{m n}:=P\left(\varphi_{m n}\right)=\left\{\begin{array}{l}0 \text { for } \varphi_{m n} \leq \xi, \\ 1 \text { for } \varphi_{m n}>\xi,\end{array}\right.$

where $\xi$ is a random number uniformly chosen from the interval $[0,1]$ and

$\varphi_{m n}=K\left(r_{m}, r_{n}, \epsilon\right) A_{n} \delta t / \Delta V$

is the probability that a particle with the radius $r_{m}$ collects one of $A_{n}$ particles with the radius $r_{n}$ within a volume $\Delta V$ during the (collection) time step $\delta t$. The collection kernel $K$ is calculated from the traditional collision efficiencies as given by Hall (1980) and includes turbulence effects by an enhancement factor for the collision efficiency by Wang and Grabowski (2009) as well as parameterizations for particle relative velocities and changes in the particle radial distribution based on Ayala et al. (2008). These turbulence effects are steered by the kinetic energy dissipation rate $\epsilon$ calculated in the LES subgrid-scale model (Riechelmann et al., 2012). The parameterizations by Ayala et al. (2008) are a direct function of $\epsilon$; the tabulated values of the enhancement factor for the collision efficiency by Wang and Grabowski (2009) are interpolated to the present value of $\epsilon$. The Eqs. (A6)-(A8) are solved using a first-order Euler method. 
Competing interests. The author declares that he has no conflict of interest.

Acknowledgements. The author thanks Siegfried Raasch and Katrin Scharf (both of the Leibniz Universität Hannover) for their helpful comments on the paper. This work has been funded by the German Research Foundation (Deutsche Forschungsgemeinschaft, DFG) under grant RA 617/27-1. Simulations have been carried out on the Cray XC-40 systems of the North-German Supercomputing Alliance (HLRN).

Edited by: Corinna Hoose

Reviewed by: Shin-ichiro Shima and one anonymous referee

\section{References}

Albrecht, B. A.: Aerosols, cloud microphysics, and fractional cloudiness, Science, 245, 1227-1230, 1989.

Andrejczuk, M., Reisner, J. M., Henson, B., Dubey, M. K., and Jeffery, C. A.: The potential impacts of pollution on a nondrizzling stratus deck: does aerosol number matter more than type?, J. Geophys. Res., 113, D19204, https://doi.org/10.1029/2007JD009445, 2008.

Ardon-Dryer, K., Huang, Y.-W., and Cziczo, D. J.: Laboratory studies of collection efficiency of sub-micrometer aerosol particles by cloud droplets on a single-droplet basis, Atmos. Chem. Phys., 15, 9159-9171, https://doi.org/10.5194/acp-15-9159-2015, 2015.

Ayala, O., Rosa, B., and Wang, L.-P.: Effects of turbulence on the geometric collision rate of sedimenting droplets. Part 2. Theory and parameterization, New J. Phys., 10, 075016, https://doi.org/10.1088/1367-2630/10/7/075016, 2008.

Böhm, J. P.: A general hydrodynamic theory for mixedphase microphysics. Part II: Collision kernels for coalescence, Atmos. Res., 27, 275-290, https://doi.org/10.1016/01698095(92)90036-A, 1992.

Chlond, A.: Locally modified version of Bott's advection scheme, Mon. Weather Rev., 122, 111-125, 1994.

Chuang, P. Y., Charlson, R. J., and Seinfeld, J. H.: Kinetic limitations on droplet formation in clouds, Nature, 390, 594-596, https://doi.org/10.1038/37576, 1997.

Devenish, B., Bartello, P., Brenguier, J.-L., Collins, L., Grabowski, W., IJzermans, R., Malinowski, S., Reeks, M., Vassilicos, J., Wang, L.-P., and Warhaft, Z.: Droplet growth in warm turbulent clouds, Q. J. Roy. Meteor. Soc., 138, 1401-1429, https://doi.org/10.1002/qj.1897, 2012.

Grabowski, W. W. and Smolarkiewicz, P. K.: Monotone finitedifference approximations to the advection-condensation problem, Mon. Weather Rev., 118, 2082-2098, 1990.

Hall, W. D.: A detailed microphysical model within a twodimensional dynamic framework: model description and preliminary results, J. Atmos. Sci., 37, 2486-2507, 1980.

Hoffmann, F.: The effect of spurious cloud edge supersaturations in Lagrangian cloud models: an analytical and numerical study, Mon. Weather Rev., 144, 107-118, https://doi.org/10.1175/MWR-D-15-0234.1, 2016.
Hoffmann, F., Raasch, S., and Noh, Y.: Entrainment of aerosols and their activation in a shallow cumulus cloud studied with a coupled LCM-LES approach, Atmos. Res., 156, 43-57, 2015.

Hoffmann, F., Noh, Y., and Raasch, S.: The route to raindrop formation in a shallow cumulus cloud simulated by a Lagrangian cloud model, J. Atmos. Sci., 74, 2125-2142, https://doi.org/10.1175/JAS-D-16-0220.1, 2017.

Holland, J. Z. and Rasmusson, E. M.: Measurement of atmospheric mass, energy, and momentum budgets over a 500-kilometer square of tropical ocean, Mon. Weather Rev., 101, 44-55, 1973.

Jaenicke, R.: Tropospheric aerosols, in: Aerosol-Cloud-Climate Interactions, edited by: Hobbs, P. V., Academic Press, San Diego, 1-27, https://doi.org/10.1016/S0074-6142(08)60210-7, 1993.

Johnson, D. B.: The role of giant and ultragiant aerosol particles in warm rain initiation, J. Atmos. Sci., 39, 448-460, 1982.

Khvorostyanov, V. I. and Curry, J. A.: Refinements to the Köhler's theory of aerosol equilibrium radii, size spectra, and droplet activation: Effects of humidity and insoluble fraction, J. Geophys. Res., 112, D05206, https://doi.org/10.1029/2006JD007672, 2007.

Köhler, H.: The nucleus in and the growth of hygroscopic droplets, T. Faraday Soc., 32, 1152-1161, 1936.

Maronga, B., Gryschka, M., Heinze, R., Hoffmann, F., KananiSühring, F., Keck, M., Ketelsen, K., Letzel, M. O., Sühring, M., and Raasch, S.: The Parallelized Large-Eddy Simulation Model (PALM) version 4.0 for atmospheric and oceanic flows: model formulation, recent developments, and future perspectives, Geosci. Model Dev., 8, 2515-2551, https://doi.org/10.5194/gmd8-2515-2015, 2015.

Naumann, A. K. and Seifert, A.: A Lagrangian drop model to study warm rain microphysical processes in shallow cumulus, J. Adv. Model. Earth Sy., 7, 1136-1154, 2015.

Nenes, A., Ghan, S., Abdul-Razzak, H., Chuang, P. Y., and Seinfeld, J. H.: Kinetic limitations on cloud droplet formation and impact on cloud albedo, Tellus B, 53, 133-149, 2001.

Riechelmann, T., Noh, Y., and Raasch, S.: A new method for largeeddy simulations of clouds with Lagrangian droplets including the effects of turbulent collision, New J. Phys., 14, 065008, https://doi.org/10.1088/1367-2630/14/6/065008, 2012.

Rogers, R., Baumgardner, D., Ethier, S., Carter, D., and Ecklund, W.: Comparison of raindrop size distributions measured by radar wind profiler and by airplane, J. Appl. Meteorol., 32, 694699, 1993.

Rogers, R. R. and Yau, M. K.: A Short Course in Cloud Physics, Pergamon Press, New York, 1989.

Shima, S.-I., Kusano, K., Kawano, A., Sugiyama, T., and Kawahara, S.: The super-droplet method for the numerical simulation of clouds and precipitation: a particle-based and probabilistic microphysics model coupled with a non-hydrostatic model, Q. J. Roy. Meteor. Soc., 135, 1307-1320, 2009.

Siebesma, A. P., Bretherton, C. S., Brown, A. R., Chlond, A., Cuxart, J., Duynkerke, P. G., Jiang, H., Khairoutdinov, M., Lewellen, D., Moeng, C.-H., Sanchez, E., Stevens, B., and Stevens, D. E.: A large eddy simulation intercomparison study of shallow cumulus convection, J. Atmos. Sci., 60, 1201-1219, 2003.

Slawinska, J., Grabowski, W. W., Pawlowska, H., and Morrison, H.: Droplet activation and mixing in large-eddy simulation of a shallow cumulus field, J. Atmos. Sci., 69, 444-462, 2012. 
Sölch, I., and Kärcher, B.: A large-eddy model for cirrus clouds with explicit aerosol and ice microphysics and Lagrangian ice particle tracking, Q. J. Roy. Meteor. Soc., 136, 2074-2093, 2010.

Twomey, S.: The nuclei of natural cloud formation part II: The supersaturation in natural clouds and the variation of cloud droplet concentration, Pure Appl. Geophys., 43, 243-249, 1959.

Twomey, S.: Pollution and the planetary albedo, Atmos. Environ., 8, 1251-1256, 1974.
Unterstrasser, S., Hoffmann, F., and Lerch, M.: Collection/aggregation algorithms in Lagrangian cloud microphysical models: rigorous evaluation in box model simulations, Geosci. Model Dev., 10, 1521-1548, https://doi.org/10.5194/gmd-101521-2017, 2017.

Wang, L.-P. and Grabowski, W. W.: The role of air turbulence in warm rain initiation, Atmos. Sci. Lett., 10, 1-8, https://doi.org/10.1002/as1.210, 2009. 